\title{
A METHOD FOR TESTING CURRENT TRANSFORMERS
}

\author{
By Francis B. Silsbee
}

CONTENTS

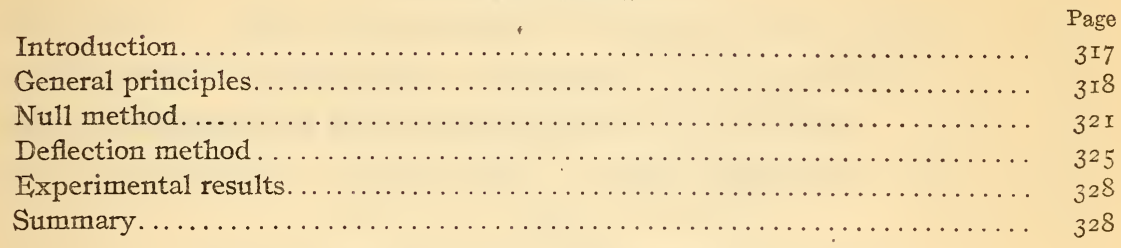

\section{INTRODUCTION}

Several precise laboratory methods are now available for the determination of the ratio and phase angle of current transformers. ${ }^{1}$ These, however, all require a considerable amount of special apparatus, such as carefully calibrated noninductive shunts and very sensitive alternating-current detectors, and are therefore not suited for use under shop or central-station conditions.

The task of comparing the constants of one transformer with those of a second transformer taken as a standard is much less difficult. The standard transformer should, of course, have the same nominal ratio, and its constants should have been determined by one of the precise laboratory methods. A method for such a comparison of two voltage transformers has been published by Brooks, ${ }^{2}$ and another method applicable to either voltage or current transformers by Agnew $^{3}$ and by Knopp. ${ }^{4}$ The method developed in this paper is somewhat analogous to the first of these and will be found rather more rapid than the second.

As in the other comparison methods the detector may be much less sensitive than in the absolute methods, and it is therefore

\footnotetext{
1 Agnew and Fitch, this Bulletin, 6, p. 28I, rg09; Electrical World, 54, p. 1042, 1909; E. Orlicl1, E. T. Z., 30, p. 435, 466, 1909; L. T. Robinson, Trans. Am. Inst. Elec. Eng., 28, p. 1005, 1909; F. A. Laws, Electrical World, 55, p. 223, 1910; Sharp and Crawford, Trans. Am. Inst. Elec. Eng., 29, p. I517, I9Io; Agnew and Silsbee, Trans. Am. Inst. Elec. Eng., :1, p. 1635, 1912; Schering and Alberti, Archiv für Elcktrotechuik, 2, p. 263 , I914.

${ }^{2}$ H. B. Brooks, this Bulletin, 10, p. 4I9, I9I4 (Scieutific Paper No. 217); Ellectrical Vorld, 62, p. 89S, $19 \mathrm{I}_{3}$.

${ }^{3}$ P. G. Agnew, this Bulletin, 11, p. 347, I9I4 (Scientific Paper No. 233).

4. A. Knopp, Electrical World, 67, p. 92, 1916.
} 
possible to use a more rugged type of instrument, such as a commercial wattmeter. Multiple-range transformers are particularly useful as standards, since they show practically proportional ratios and identical phase angles on the various primary connections. Two or three such transformers, the ratio and phase angle of which have been accurately determined, suffice for testing a considerable range of transformers.

\section{GENERAL PRINCIPLES}

The principle of the method is illustrated by Fig. I. $S$ and $X$ are the standard and the unknown transformer, respectively. The primary windings are connected in series and supplied with current from a suitable source. The secondary windings are also

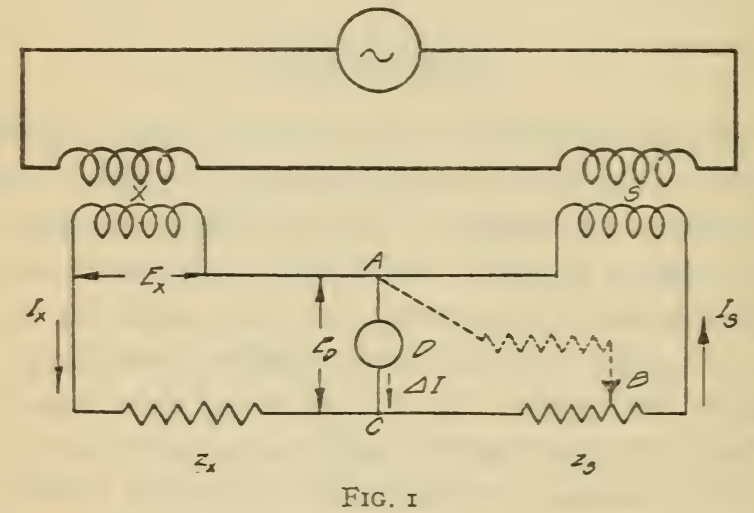

connected in series, with such polarity that both tend to send current in the same direction, and any desired impedance loads such as $z_{\mathrm{s}}$ and $z_{\mathrm{x}}$ complete the circuit. A suitable detector $D$ is then connected so as to bridge across between the transformers.

It is evident that the current $\Delta I$ through the detector (which we shall assume for the present to have a negligible impedance) is necessarily equal to the vector difference of the secondary currents $I_{\mathrm{s}}$ and $I_{\mathrm{I}}$ of the transformers. Consequently, if the magnitude and phase of $\Delta I$ are measured, the difference in performance of the two transformers can be computed. This measurement of $\Delta I$ may be made either directly by using as a detector one winding of a separately excited wattmeter, as is described below as the "Deflection method," or the measurement may be made indirectly by using an additional compensating circuit between $A$ and $B$, as indicated by the dotted lines. By proper arrangement of the impedances all of $\Delta I$ may be made to flow in this 
compensating circuit, and the current in the detector reduced to zero. This is described below as the "Null method." There are, of course, a great many other possible arrangements for measuring $\Delta I$, but the two here described in detail will be found the most convenient.

The performance of a current transformer depends to a considerable extent upon the impedance of the apparatus and wiring connected in the secondary circuit. It is therefore of importance to determine what impedance is introduced into the circuit of each transformer by the arrangement of circuits shown in Fig. I. Now transformer $X$ is carrying a current $I_{\mathbf{x}}$ at a terminal voltage $E_{\mathrm{X}}$, and the equivalent impedance load is

$$
Z_{\mathrm{x}}=\frac{E_{\mathrm{x}}}{I_{\mathrm{x}}}=\frac{I_{\mathrm{x}} z_{\mathrm{x}}-E_{\mathrm{d}}}{I_{\mathrm{x}}}=z_{\mathrm{x}}-\frac{E_{\mathrm{d}}}{I_{\mathrm{x}}}
$$

(vectorially)

In the deflection method $E_{\mathrm{d}}$ (the voltage between the terminals of the detector) is

And hence

$$
E_{\mathrm{d}}=\Delta I z_{\mathrm{d}}
$$

$$
Z_{\mathrm{x}}=z_{\mathrm{x}}-z_{\mathrm{d}} \frac{\Delta I}{I_{\mathrm{x}}}
$$

(vectorially)

and similarly

$$
Z_{\mathrm{s}}=z_{\mathrm{s}}+z_{\mathrm{d}} \frac{\Delta I}{I_{\mathrm{s}}}
$$

It is therefore evident that when the secondary currents are nearly equal, so that $\frac{\Delta I}{I_{\mathrm{s}}}$ is very small, a detector of considerable impedance and therefore of high-current sensitivity may be used. On the other hand, when $\frac{\Delta I}{I_{\mathrm{s}}}$ is large, then the use of a detector of high impedance may shift the load from the transformer of higher ratio to that having the lower and thus make the difference in ratio appreciably less than the correct value.

In the null method, on the other hand, $E_{\mathrm{d}}$ is zero when a balance is reached, so that

$$
Z_{\mathrm{x}}=z_{\mathrm{x}}
$$

and the performance of each transformer is the same as if the other transformer were replaced by a short-circuiting link across $A C$. Although very evident theoretically, this fact was also tested experimentally, and the behavior of the transformer was found to be the same as when the detector was replaced by a wire of negligible resistance. 
The principal limitation on the sensitivity attainable in any method of testing current transformers is due to the condition that the measuring circuit must not affect the performance of the transformer. This means that the measuring apparatus must not increase the equivalent connected secondary load by more than a certain resistance which we may denote by $r$.

The gain in sensitivity of a comparison method, such as is described in this paper over an absolute method, is seen by comparing the power available for operating the detector in the two cases. In the usual absolute method the secondary current $I$ is passed through a noninductive resistance which is preferably equal to $r$. The voltage drop Ir is balanced by the drop due to the primary current flowing through a proportionately smaller resistance. With such an arrangement the impedance of the circuit external to the detector is approximately $r$, and for the maximum sensitivity the detector itself should also have this same impedance. If under these conditions the secondary current should differ by a small amount $\delta I$ from that required for an exact balance, then the unbalanced voltage acting in the detector circuit will be only $\delta I r$. Since the resistance of the complete circuit is $2 r$, the current flowing in the detector will be $\frac{\delta I}{2}$, and the voltage across the detector will be $\frac{\delta I r}{2}$. Consequently the power available is $\frac{\overline{\delta I}^{2}}{4}$.

On the other hand, in the case of the deflection method outlined in this paper, the total difference $\Delta I$ between the two secondary currents flows through the detector and produces a voltage at its terminals equal to $\Delta I z_{\mathrm{d}}$. Here $z_{\mathrm{d}}$ is the impedance of the detector, and is limited by the fact that this voltage $\Delta I z_{\mathrm{d}}$ must not exceed the permissible value $I r$ for the largest value of $\Delta I$. Consequently, we have $z_{\mathrm{d}} \equiv \frac{I}{\Delta I} r$ and if we use a detector having this impedance, the volt amperes available for a difference $\delta I$ in the currents is $\overline{\delta I}^{2} \frac{I}{\Delta I}$. Since the two transformers will usually differ by only a few per cent the factor $\frac{I}{\Delta I}$ is fairly large and the detector required may be 50 or roo times less sensitive than in the precise laboratory methods. It is this relation which brings the method within the range of sensitivity obtainable with commercial pivoted instruments. 
The connections of what is probably the most satisfactory form of null method based on the general arrangement outlined above are given in Fig. 2. $A B C D$ is a slide wire of about 0.2 ohm total resistance. $M$ is a mutual inductance of about 600 microhenrys, the primary of which can carry 5 amperes without excessive heating. $\quad r_{1}$ is a resistance of about $30 \mathrm{ohms}$, preferably capable of being set at several other values down to 2 ohms. As the value of $r_{1}$ appears in the denominator in the equations below it is convenient to have the total resistance between $F$ and $C$, including the resistance of the secondary winding of $M$, an exact integer for each setting of $r_{1}$. $M, r_{1}$ and $r_{2}$ need be calibrated only to the per cent accuracy which is desired in the difference of the ratios; that is, to about I per cent.

The detector shown in the figure is a separately excited electrodynamometer instrument. A commercial wattmeter of $10 \mathrm{w}$ current range may be adapted for this work by bringing out taps directly from the moving coil without using any of the series resistance. The moving coil is connected as shown and the current coil excited by its full rated current in either of two phases, which preferably are in quadrature, through the double-throw switch $G$. Any other form of alternating-current detector sensitive to 0.00005 ampere might be used. ${ }^{5}$

As a precaution a ro-ampere ammeter should be connected in parallel with the detector, so that the transformers will not be damaged if they have inadvertently been connected in opposition instead of aiding. If on closing the circuit this ammeter shows no appreciable current the polarity of the transformers is correct, and the ammeter should then be disconnected.

The procedure is to adjust the position of the slider $C$ and the value of the mutual inductance $M$ until no deflection is obtained on closing $G$ in either direction. When a balance is thus obtained all of the differential current $\Delta I$ is flowing through $r_{1}$, and also the difference of potential between points $B$ and $F$ is zero. Consequently, the voltage drops between $C$ and $F$ and between $C$ and $B$ must be equal and in phase with one another. From this relation the differences in the ratios and phase angles of the two transformers may be computed.

\footnotetext{
5 A new type of vibration galvanometer has been recently developed by Agnew which is very well suited for this work. A description of this sensitive yet rugged instrument is to be published shortly in this Bulletin.
} 
If $R_{\mathrm{a}}$ and $R_{\mathrm{x}}$ are the ratios of the transformers $S$ and $X$, respectively, and $\alpha_{s}$ and $\alpha_{x}$ are the corresponding phase angles, then we have ${ }^{6}$ for the case where the slider $C$ is to the right of $B$ (Fig. 2)

$$
\frac{R_{\mathrm{x}}}{R_{\mathrm{B}}}=\mathrm{I}+a-\frac{b^{2}}{2}-b c
$$

and

$$
\tan \left(\alpha_{\mathbf{x}}-\alpha_{\mathbf{s}}\right)=b+a c
$$

The equations given below may be deduced as follows: For the connections as drawn in Fig. 2

we have

$$
\Delta I=I_{0}-I_{\mathrm{x}}
$$

and

$$
\Delta I\left(r_{1}+j \omega L_{1}\right)+I_{s} \omega M=I_{2} r_{2}
$$

by Kirchhoff's laws, where the currents are to be regarded as vector quantities.

Hence, eliminating $\Delta I$, we get

$$
\begin{gathered}
I_{3}\left\{n+j \omega\left(L_{1}+M\right\}=I_{\mathrm{x}}\left(r_{1}+r_{s}+i \omega L_{1}\right)\right. \\
\frac{I_{3}}{I_{x}}=\frac{1+\frac{r_{0}}{r_{1}}+j \omega \frac{L_{1}}{r_{1}}}{1+i\left(\frac{\omega L_{1}}{r_{1}}+\frac{\omega M}{r_{1}}\right) .}
\end{gathered}
$$

Substituting

$$
a=\frac{r_{2}}{r_{1}}, b=\frac{\omega \cdot M}{r_{1}}, c=\frac{\omega L_{1}}{r_{1}}
$$

$$
\frac{I_{b}}{I_{x}}=\frac{1+a+j c}{I+j(b+c)}=1+a-b c-b^{2} \ldots+j(-b-a c-a b \ldots)
$$

This quotient must now be determined in terms of the current ratios and phase angles of the two transformers. The ratio of a current transiormer is simply the ratio of the magnitude of the primary current, $I_{\mathrm{p}}$, to that of the secondary. But since we are now regarding the currents as vector quantities, we must write for the ratios

$$
\begin{aligned}
& R_{\mathrm{b}}=\frac{I_{\mathrm{P}}}{I_{\mathrm{g}}}\left(\cos \alpha_{\mathrm{s}}+j \sin \alpha_{\mathrm{s}}\right) \\
& R_{\mathrm{x}}=\frac{I_{\mathrm{P}}}{I_{\mathrm{x}}}\left(\cos \alpha_{\mathrm{x}}+j \sin \alpha_{\mathrm{x}}\right)
\end{aligned}
$$

where the complex factors in the parentheses have been introduced to make the ratios themselves $(R$, and $R_{\mathrm{x}}$ ) simple numbers instead of vector quantities. (The complex factor in each case merely tums the vector $I_{\mathrm{p}}$ through the angle $\alpha$, into coincidence with the secondary current.) It is to be noted that this assumes that the phase angle, $\alpha$, is positive when the reversed secondary current leads the primary current.

From (6) and (7) we have

$$
\frac{R_{x}\left(\cos \alpha_{s}+j \sin \alpha_{8}\right)}{R_{s}\left(\cos \alpha_{x}+i \sin \alpha_{x}\right)}=\frac{I_{s}}{I_{x}}
$$

or rationalizing

$$
\frac{R_{x}}{R_{6}}\left\{\cos \left(\alpha_{3}-\alpha_{x}\right)+j \sin \left(\alpha_{s}-\alpha_{x}\right)\right\}=\frac{I_{2}}{l_{x}}
$$

Equating (5) and (9) and separating the real and imaginary terms, we get

$$
\frac{R_{x}}{K_{B}} \cos \left(\alpha_{u}-\alpha_{x}\right)=1+a-b c-b^{2} .
$$

and

$$
\frac{R_{\mathrm{x}}}{R_{\mathrm{s}}} \sin \alpha\left({ }_{0}-\alpha_{\mathrm{x}}\right)=-b-a c-a b \ldots
$$

Solving these equations simultaneously, we have

$$
\begin{aligned}
& R_{\mathrm{x}} \\
& R_{\mathrm{a}}
\end{aligned}=\left\{\left(\mathrm{x}+a-b c-b^{2}\right)^{2}+(-b-a c-a b)^{2}\right\}^{1 / 2}=1+a-\frac{b^{2}}{2}-b c
$$

and

$$
\tan \left(\alpha_{x}-\alpha_{s}\right)=b+a c \ldots
$$

which are the formulas desired.

The deduction for the case when the slide $C$ is to the left of $B$ (Fig. 2 ) is similar to the above with slight differences in the second-order terms. 
For the case where $C$ is on the same side of $B$ as transformer $X$, then

$$
\frac{R_{\mathrm{x}}}{R_{\mathrm{s}}}=\mathrm{I}-a+\cdot a^{2}-\frac{b^{2}}{2}-b c
$$

$$
\tan \left(\alpha_{\mathrm{x}}-\alpha_{\mathrm{s}}\right)=b-a c-a b
$$

where

$$
a=\frac{r_{2}}{r_{1}}, \quad b=\frac{\omega M}{r_{1}}, \quad c=\frac{\omega L_{1}}{r_{1}}
$$

and

$\omega=2 \pi \times$ frequency.

$r_{1}=$ the total resistance between $C$ and $H$ through $r_{1}$ and $M$ (Fig. 2), in ohms.

$r_{2}=$ the resistance of the slide wire between $B$ and $C$, in ohms.

$L_{1}=$ the self-inductance of the secondary coil of the mutual inductance $M$, in henrys.

$M=$ the value of the mutual inductance, in henrys. (This is to be taken as positive if $\alpha_{\mathrm{z}}$ is greater than $\alpha_{\mathrm{s}}$ as shown by the test described below.)

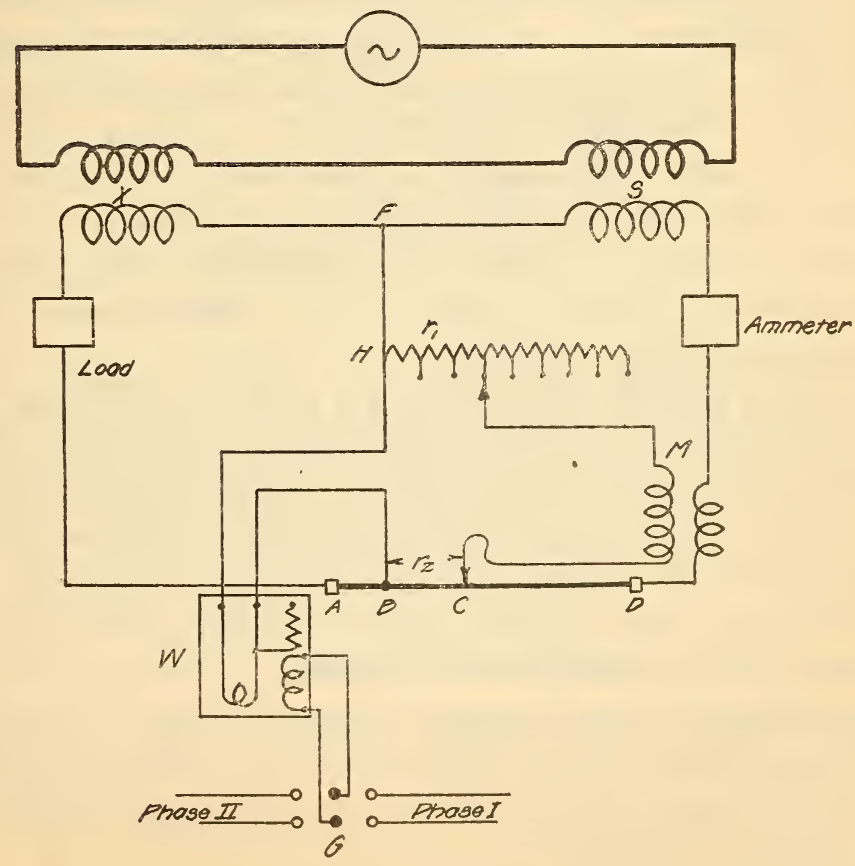

FIG. 2 
The second-order terms are usually very small and to an accuracy of a tenth per cent we have in either case

$$
\begin{aligned}
& R_{\mathrm{x}}=R_{\mathrm{s}}(\mathrm{I} \pm a) \\
& \alpha_{\mathrm{x}}=\alpha_{\mathrm{s}}+343^{8} b
\end{aligned}
$$

if the angles are expressed in minutes.

If the balance is obtained with the slider between $B$ and $D$ (Fig. 2), then (provided the second-order terms are less than $a$ ), the standard transformer is supplying the greater secondary current and consequently has the smalier ratio. The question which secondary current leads the primary current by the greater angle can best be determined once for all by adding some resistance to the load on transformer $X$. This will always make $I_{x}$ lead more than before and if the value of $M$ required for a balance is increased then $I_{\Sigma}$ was leading originally and $M$ and $b$ are to be taken as positive with this connection of the mutual inductance.

In the case when the slider $C$ is to the right of $B$ (Fig. 2) and the part $A B$ of the slide wire is omitted, then the measuring circuits introduce no impedance whatever into the equivalent secondary circuit of transformer $X$. It is therefore possible by this arrangement to test a current transformer under the condition of zero external load. The load on transformer $S$ or, in case $C$ is to the left of $B$, on both transformers, is practically the same as the impedance of the slide wire and the primary winding of $M$. The slight change in this, due to the fact that $r_{1}$ in effect shunts $r_{2}$, is of the order $a r_{2}$ and its effect on the performance of either transformer is entirely negligible.

It is evident that if $S$ always had a smaller ratio than $X$ the slider would always be on the standard side of $B$ and the part $A B$ of the slide wire would be unnecessary. This arrangement is very desirable since it does away with the necessity of allowing for the resistance of $A B$ in making the total impedance load on transformer $X$ equal to the desired value. The ratio of the standard transformer could be reduced by omitting a few turns from the secondary winding in the process of manufacture. In completed transformers of the hole type this can still be done by passing a few secondary turns through the hole in a direction to oppose the main secondary winding.

Since at the point of balance no current flows through the moving coil of the detector the self-inductance of this coil has no effect on the results. The mutual inductance between the two 
coils of the wattmeter, however, does produce an electromotive force between the points $B$ and $F$ even when no current flows and therefore affects the setting. This source of error can easily be eliminated by shifting the position of the control springs so that the normal zero of the instrument corresponds to the position of zero mutual inductance. ${ }^{7}$

If the current in Phase I of the exciting circuit is in phase with the current in the transformers and Phase II is $90^{\circ}$ from this, then the slider $C$ may be adjusted with $G$ closed to the right, and $M$ with $G$ to the left, and the two settings will be independent of each other. It is not at all necessary, however, that these phase relations be exact and Phase II may be $60^{\circ}$ from Phase I,

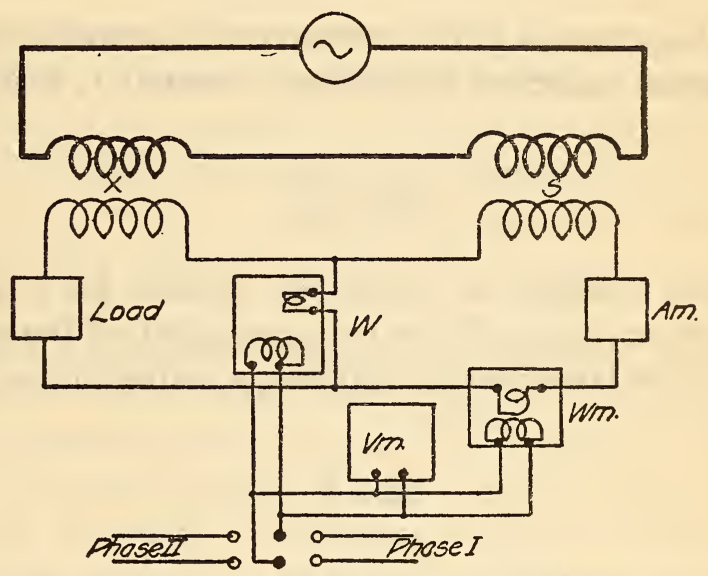

FIG. 3

as in a 3 -phase system. If a polyphase supply is not available, current for Phase I may be drawn through a lamp bank and Phase II from the same supply through a reactance coil or transformer winding. With this arrangement it is well not to work the iron of the core much above its normal rating or the harmonics in the exciting current will become excessive.

\section{DEFLECTION METHOD}

In cases where a variable mutual inductance and slide wire are not available, but a polyphase supply is at hand, the deflection method (the connections for which are shown in Fig. 3) may

7 This position can be determined by short-circuiting the moving coil (or better still by connecting it to a large inductance of low resistance, such as the 220 -volt winding of a transformer) and passing full-rated current through the fixed coil. Under these conditions the pointer deflects toward the position of zero mutual inductance, and by suitably moving the control springs the desired position can soon be located. An alternative method is to connect a telephone receiver to the moving coil and pass full-rated current through the fixed coil. The springs can then be adjusted until no sound is lieard in the telephone.

$$
59467^{\circ}-18-11
$$


be used. The detector $W$ in this method must be of the dynamometer type, and arranged so that one coil may be supplied from two circuits, one giving a current in phase with the current in the transformers and the other giving a current having a known phase relation, preferably quadrature with the former. If $D_{1}$ and $D_{\mathrm{q}}$ are the deflections in divisions, observed with the excitation in phase, and in quadrature, respectively, we have

$$
\begin{gathered}
R_{\mathbf{x}}=\mathrm{I}+\frac{K D_{1}}{I_{\mathrm{s}}} \\
R_{\mathrm{s}} \\
\tan \left(\alpha_{\mathrm{x}}-\alpha_{\mathrm{s}}\right)=\frac{K D_{\mathrm{q}}}{I_{\mathrm{s}}}, \text { approximately, }
\end{gathered}
$$

where $K$ is the constant of the instrument in amperes per division.

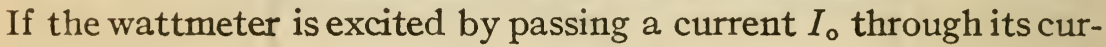
rent coil,

$$
K=\frac{k}{I_{\mathrm{o}} R_{\nabla}}
$$

where $k$ is the constant in watts per division for a given range and $R_{\nabla}$ is the resistance of the voltage circuit of the same range. If the wattmeter is excited by applying a voltage $E_{\mathrm{o}}$ to the voltage circuit, then

$$
K=\frac{k}{E_{\mathrm{o}}}
$$

In case the second phase available as a source of excitation is not in quadrature with the first, but gives exciting current which lags behind the current in the transformers by an angle $\theta$ and produces a corresponding deflection $D_{\theta}$, then we have

$$
\begin{gathered}
\frac{R_{\mathrm{x}}}{R_{\mathrm{s}}}=\mathrm{I}+\frac{K D_{\mathrm{i}}}{I_{\mathrm{s}}} \\
\tan \left(\alpha_{\mathrm{x}}-\alpha_{\mathrm{s}}\right)=\frac{K}{I_{\mathrm{s}}}\left(\frac{D_{\theta}-D_{\mathrm{i}} \cos \theta}{\sin \theta}\right)
\end{gathered}
$$

As before, the question which secondary current is the greater and which leads the primary current by a greater angle can best be answered by changing the load on one transformer and noting the effect on the deflections, remembering that an increase in secondary resistance makes the secondary current smaller and also makes it lead the primary current more. 
The principal limitation of this method is, as mentioned above, the effect of the impedance of the detector in shifting the load from one transformer to the other. The change in the equivalent impedance load on either transformer due to this is given by

$$
\Delta Z=\frac{z_{\mathrm{d}} \Delta I}{I_{\mathrm{s}}}=\frac{z_{\mathrm{d}} K \sqrt{D_{\mathrm{i}}^{2}+D_{\mathrm{q}}^{2}}}{I_{\mathrm{s}}}
$$

Since this varies with the deflection it is impracticable to allow for this impedance in arranging the secondary loads on the transformers and it is necessary to reduce $\Delta Z$ not to a comparatively small and definite value, as in the null method, but to a value which can be entirely neglected. If the moving coil of a commercial wattmeter is used as a detector, it must therefore be shunted by a rather low resistance and the sensitivity correspondingly reduced. The current circuit of a I-ampere wattmeter has about the desired impedance and will be found satisfactory as a detector for this work. The use of the voltage circuit for the excitation is also convenient, since it avoids the necessity of a separate resistance.

The mutual inductance between the coils also introduces an equivalent load on the transformers, but if over the part of the scale used the coil is reasonably near the position of zero mutual inductance, this error will be less than that due to the impedance.

As a specific example of the various factors entering into the choice of a detector, let us consider the commercial wattmeter used in the experiments described below, which has the following constants:

\begin{tabular}{|c|c|}
\hline Nominal current.................... & 2. amperes. \\
\hline 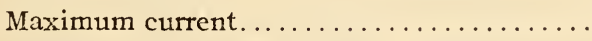 & 3. amperes. \\
\hline Nominal voltage. ...... & 75. volts. \\
\hline Maximum voltage $\ldots \ldots \ldots \ldots \ldots \ldots \ldots \ldots$ & I IO. volts. \\
\hline Resistance of fixed coil .................. & $0.27 \mathrm{ohm}$. \\
\hline Resistance of moving coil ............... & 42. ohms. \\
\hline Resistance of voltage circuit............. & I, I78. ohms. \\
\hline Watts per division.................... & \\
\hline
\end{tabular}

When this instrument is used with excitation on the voltage circuit we have

$$
\begin{aligned}
& K=\frac{0.5}{\mathrm{IIO}}=4.5 \times 1 \mathrm{O}^{-3} \text { amperes per division, } \\
& z_{\mathrm{d}}=0.27 \mathrm{ohm}, \text { approximately. }
\end{aligned}
$$


When used with excitation on the current circuit the moving coil must be shunted to give a resistance of about $\mathbf{I}$ ohm so that

$$
\begin{aligned}
& K=\frac{0.5}{3 \times 1 \mathrm{I} / \mathrm{S}} \times \frac{\ddagger^{2}}{\mathrm{I}}=6.0 \times 10^{-3} \text { amperes per division, } \\
& z_{\mathrm{d}}=\mathrm{I} \text { ohm, approximately. }
\end{aligned}
$$

If the two transformers differ by i per cent in current, then at the 5 -ampere point we have in the first case the deflection

$$
D=8.3 \text { divisions }
$$

and

$$
\Delta Z=0.003 \text { ohm, }
$$

and in the second case

and

$$
D=\text { I I . divisions }
$$

$\Delta Z=0.0 \mathrm{I}$ ohm.

A further error arises if the exciting voltages are not in the correct phase. The magnitude of this effect varies greatly with the difference in constants of the two transformers. If this difference is I per cent in ratio and 30 minutes in phase angle, a shift in phase of 7 degrees in the exciting voltage will produce an error of about o.I per cent in ratio and 3 minutes in angle. An auxiliary 5-ampere wattmeter connected as shown at $W m$ in Fig. 3 is a convenient means of determining these phase relations.

\section{EXPERIMENTAL RESULTS}

Both of the methods described above have been tried experimentally and the results are plotted on a large scale in Fig. 4. Curves $A$ and $B$ give the ratio factor and phase angle, respectively, of a 25-ampere, 40 volt-ampere portable current transformer at 60 cycles. The curve is drawn through values obtained by a precise laboratory method. The crosses are points observed by the null method using as a standard a similar transformer carrying considerably less load. Curves $C$ and $D$ give the ratio factor and phase angle of another transforner which was tested by the deflection method. As before, the curves are drawn through the points obtained by a precise laboratory method and the circles show the values obtained by the method here proposed.

\section{SUMMARY}

A general method has been outlined for the determination of the ratio and phase angle of current transformers in terms of the constants of previously calibrated standard transformers of the 
same nominal ratio. It has been shown that such methods are essentially more sensitive, or conversely may be used with much less sensitive instruments, than the laboratory methods now in use for the absolute determination of the ratio and phase angle of a single transformer. Two of the most convenient of the many possible modifications of the general method are described in

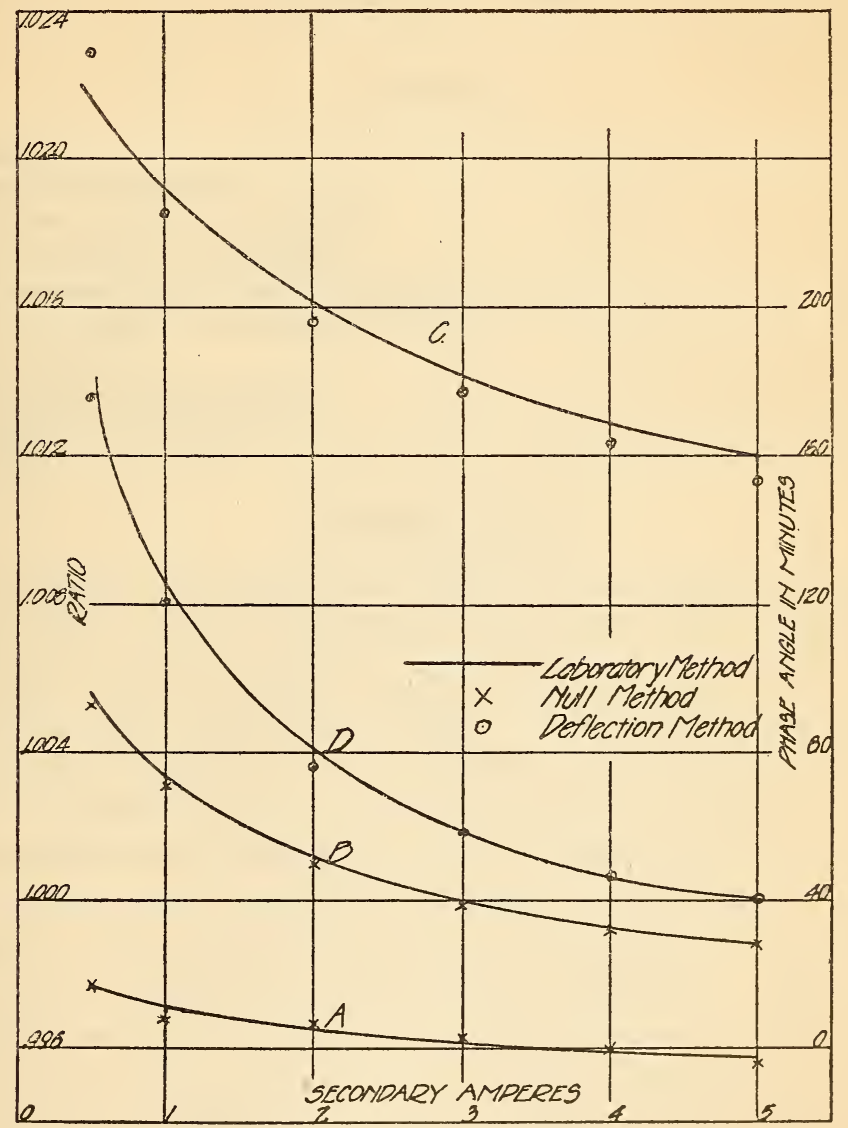

FIG. 4

detail. The experimental results obtained on two pairs of transformers by these methods agree very satisfactorily with the values obtained by absolute methods. It is hoped that the methods will be found useful in commercial plants where delicate laboratory equipment is not available, and where large numbers of transformers must be tested rapidly and with moderate accuracy.

WASHINGTON, July I, I 917. 